\title{
Influence of Temperature on Gamma-Oryzanol Stability of Edible Rice Bran Oil during Heating
}

\author{
A. Srisaipet and M. Nuddagul
}

\begin{abstract}
Rice bran oil (RBO) presents a large amount of nutraceutical compounds such as gamma-oryzanol, a complex mixture of ferulate esters with sterol. It shows high ability to reduce cholesterol absorption and inhibition oxidation reaction. This research evaluates the stability of $\gamma$-oryzanol present in refined $\mathrm{RBO}$ by heating the oil under different temperatures. From the data, the concentration of $\gamma$-oryzanol are slightly changing at temperature of the oil higher than $120{ }^{\circ} \mathrm{c}$ for isopropanol, butanol and ethyl acetate as dissolve solvent. While hexane showed balanced of $\boldsymbol{\gamma}$-oryzanol trend in all of studying temperature which is affected by the interference of the oil matrix. Thus isopropanol had been suggested for accuracy solvent for $\gamma$-oryzanol determination. Further, we found that that the acid value of RBO depends on temperature of heated oil and $\gamma$-oryzanol content.
\end{abstract}

Index Terms-Rice bran oil, gamma-oryzanol, organic solvent, heating stability.

\section{INTRODUCTION}

Rice (Oryza sativa) is the most popular food crop in Asia and Thailand, due to it is main food for the population in this area. Rice bran, a by-product which is obtained from the rice milling processes, contains about $8-11 \%(\mathrm{w} / \mathrm{w})$ of the grain and compost of $16-22 \%$ of lipid. Rice bran can be used as feed or as a major source of rice bran oil (RBO) [1], [2].

Rice bran oil (RBO) is widely popular edible oil in east Asia countries such as China, Korea, Japan, Taiwan and Thailand. India and Thailand are the most important procedures of RBO and together sum over 225,000 metric tons of RBO per years [3]. The utilization of RBO has increased considerable in Western countries due to its well-known for wealth of phytochemical and it is very stable in at high temperature [4]. Gamma-oryzanol ( $\gamma$-Oryzanol) derivatives in particular are found in only a very limited number if oils and it is the most important phytochemical components in RBO which is present at 13-20 times (w/w) greater content in rice bran than total tocopherols and tocotrienols [5]. $\gamma$-Oryzanol is a mixture of whole group of ferulic acid ester of triterprene alcohols and phytosterol [6]. $\gamma$-Oryzanol is mainly composed of four ester are cycloartanyl ferulate, 24-methylene cycloartany ferulate, $\beta$-sitosteryl ferulate and campesteryl ferulate [7]. It has been reported to lower blood cholesterol levels in humans and animals [8], [9], anti-inflammatory [10], anti-tumor activities [11], and

Manuscript received November 6, 2013; revised January 15, 2014. This work was supported by Department of Chemistry, Faculty of Science Maejo University, Chiang Mai, Thailand.

The authors are with the Department of Chemistry, Faculty of Science, Maejo University, Chiang Mai, Thailand (email: anakhaorn@mju.ac.th). including antioxidant [7], [12], [13]. In addition to these putative health benefits, the antioxidants of RBO have a potential uses as additives to improve the storage stability of foods [14], [15].

Rice bran oil has a high nutritional value and it is very appealing as specialty oil in niche market. It is used as edible oil in many Thailand households. In a cooking process, the oil is heated at high temperature although the cooking time in households is shorter period than industrial process. However losses of phytochemicals in the oil can not be avoided.

The most commonly used technique for characterization is of $\gamma$-oryzanol in RBO is HPLC and the technique for determination $\gamma$-oryzanol content is UV spectrophotometry [16] because it is very simple, rapid and easy operating.

The objective of this study was to evaluate the stability of $\gamma$-oryzanol present in refined RBO when heated under different temperatures. The UV-spectrophotometry method is used to quantify the $\gamma$-oryzanol content in the RBO. Thereby, we simulate cooking condition in households which is shorter time and lower temperature than industrial process.

\section{MATERIALS AND METHOD}

\section{A. Materials}

The four brands (Chim, King, King (high oryzanol) and Alpha-1) of commercial rice bran oil were purchased from local supermarket. Gamma- oryzanol was supplied by the Vegetable Oil Refinery (Bangkok, Thailand). Butanol, Ethyl acetate, Isopropanol and hexane in analytical grade which purchase from LabScan (Bangkok, Thailand) were used as solvent for spectrophotometric technique. The bran was dried at $105{ }^{\circ} \mathrm{c}$ until the weight constant.

\section{B. Studying of Solvent Effect on the Accuracy of Gamma-Oryzanol Determination}

Exactly $1.0 \mathrm{~g}$ dried bran was weight into screw cap test tube and added $4 \mathrm{ml}$ of solvent (hexane, ethy acetate, isopropanol and butanol). Extraction was done by mixing the substances for 30 minutes at room temperature $\left(27^{\circ} \mathrm{C}\right)$ and incubated overnight. Centrifugation was used for separate the bran from miscella for 5 minutes at 3,000 rpm. Supernatants was collected and recorded for $\gamma$-oryzanol absorption spectra. The four brand of commercial RBO were dissolved in solvent and the $\gamma$-oryzanol in the oil absorbance was done by using a U-100 UV-vis spectrophotometer (HITASHI, Japan). Quantification of $\gamma$-oryzanol in solvent was determined by standard curve.

C. Stability of Gamma-Oryzanol in Heated Rice Bran Oil 
Rice bran oil was submitted to heating in oil bath at 80,100 , 120, 140, 160 and $180{ }^{\circ} \mathrm{c}$ for 20 minutes without air circulation and protection from light. The RBO samples were taken from the heating bath and were immediately cooled in ice bath to stop the reaction. The sample was dissolved with the solvent and the absorbance spectra of sample were detected at optimum wavelength.

\section{Determination of Acid Value}

Acid value (AV) of RBO was applied from AOCS Official method Method (AOCS, 1999). The oil sample was dissolved in isopropanol/ toluene mix (1:1), followed by addition of phenolphthalein indicator (1\% in isopropanol) and neutralization with base. The sample is determined quantitatively by titration with $0.1 \mathrm{~N}$ potassium hydroxide. The AV values are express as $\mathrm{mg} \mathrm{KOH/g}$ of sample.

\section{RESULT AND DISCUSSION}

\section{A. Studying of Solvent Effect on the Accuracy of Gamma-Oryzanol Determination}

Recently, the reputedly solvent for high oil extraction is hexane [17]. Therefore, the other solvent types were compared in accuracy determination of $\gamma$-oryzanol content. The all of extraction solvents are hexane, ethyl acetate, isopropanol and butanol. The maximum wavelengths $\left(\lambda_{\max }\right)$ and standard calibration of $\gamma$-oryzanol analysis in different were summarized in Table I which is closely to a previous reported [18]. The good linearly with correlation coefficients $\left(R^{2}\right)$ showed greater than 0.995 , in the range studied.

TABLE I: MAXIMUM WAVELENGTHS $\left(\lambda_{\text {MAX }}\right)$ AND STANDARD CALIBRATION FOR $\gamma$-ORYZANOL ANALYSIS IN DIFFERENT ORGANIC SOLVENT

\begin{tabular}{lcccc}
\hline \hline \multirow{2}{*}{ Solvent } & \multicolumn{2}{c}{$\lambda_{\max }(\mathrm{nm})$} & \multicolumn{2}{c}{ Calibration curves } \\
\cline { 2 - 5 } & Ref. & Experimental & $\begin{array}{l}\text { Regression } \\
\text { Equation* }\end{array}$ & $R^{2}$ \\
\hline Hexane & 314 & 315 & $y=0.0359 x$ & 0.997 \\
Ethyl & 320 & 320 & $y=0.0386 x$ & 0.999 \\
acetate & 326 & 327.5 & $y=0.0393 x$ & 0.996 \\
Isopropanol & 326 & 328 & $y=0.0350 x$ & 0.995 \\
Butanol & & & & \\
\hline \hline * $y=$ absorbance, $x=$ concentration $(\mathrm{ppm})$ & &
\end{tabular}

Rapid equilibrium extraction methods have been used to extracted oil from rice bran [19], [20]. Fig. 1 shows the concentrations of $\gamma$-oryzanol in the rice bran extracted by different organic solvent. The $\gamma$-oryzanol concentration range were found from 6,400 to $6,900 \mathrm{ppm}$ which is nearly value in both of low polar (hexane and ethyl acetate) and high polar (isopropanol and butanol) solvent extracted. HU et al., 1996 and Nanua et al., 2000 [15], [21] reported the more polar solvents, isopropanol or isopropanol: hexane $(1: 1 \mathrm{v} / \mathrm{v})$, extracted more vitamin E homologs or $\gamma$-oryzanol, respectively, from rice bran than hexane. Chen and Bergman 2005 [22] noted that the hydroxyl groups on the benzene ring of ferulate esters might be interacted with alcohol functional group of isopropanol, rendering more liberations of $\gamma$-oryzanol during extraction. Zigoneanu et al., 2008 [23] proposed that no significant change in amount of $\alpha$-tocopherol dissolved in isopropanol and hexane during the processes of microwave and solvent extractions in heating and evaporation at $40{ }^{\circ} \mathrm{c}$. Proctor and Bowen, 1996 [19] suggested that isopropanol was used as the extraction solvent in comparison with hexane, the same amount of oil was extracted from rice bran. In addition, the polarity of butanol is very closely with isopropanol then its extractability was nearly isopropanol. These polar solvent are miscible, water on the rice bran surface can not retard the penetration of these solvents compared with polarity solvent. Thereby, Qiao et al., 2005 [24] remarked that the different degree of coupled transport of isopropanol and butanol had affected to physicochemical properties of penetrants and membrane materials. For these reasons, these solvents include hexane, ethyl acetate, isopropanol and butanol will be used to study the $\gamma$-oryzanol concentration in commercial edible RBO.

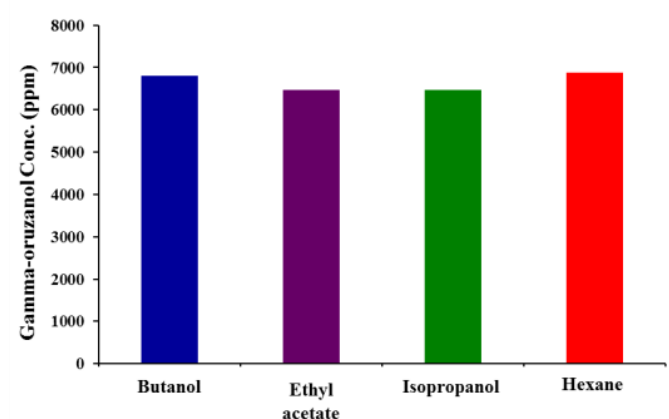

Fig. 1. The Gamma-oryzanol concentration in crude RBO solution which extracted by organic solvent.

\section{B. Quantification of Gamma-Oryzanol in Commercial Rice Bran Oil}

Due to the different $\gamma$-oryzanol content in each commercial RBO brand. The four brands (Chim, King, King (high oryzanol) and Alpha-1) of commercial RBO were selected to determine $\gamma$-oryzanol concentration in the oil. The $\gamma$-oryzanol content were label for higher than 2000, 4000 and 5,000 ppm for King, King (high oryzanol) and Alpha-1 brands, respectively and it was not labelled for Chim brand. The $\gamma$-oryzanol content analyzed by dissolving butanol, ethyl acetate, isopropanol and hexane at $27^{\circ} \mathrm{C}$ is presented in Fig. 2. We observed that detection of $\gamma$-oryzanol in hexane show highest value in overall solvent in all RBO brand except the content in Alpha 1 brand which dissolved in butanol. However, the $\gamma$-oryzanol detection via dissolved in four solvent were in good agreement with the labelled value in all of RBO brand.

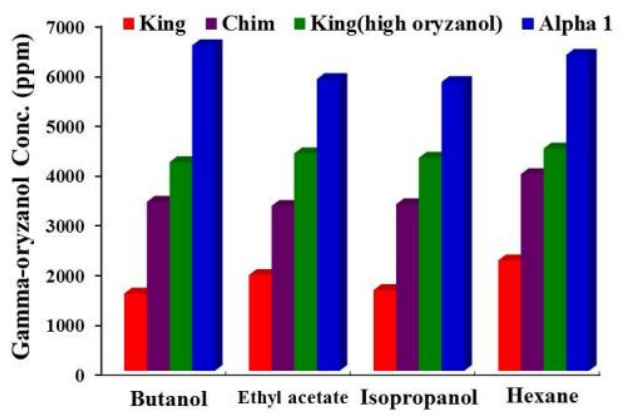

Fig. 2. The quantification of Gamma-oryzanol in commercial RBO analyzation via different solvent.

Varition of $\gamma$-oryzanol concentration analyzation with different temperature and solvent is exhibited in Fig. 3. When 
isopropanol, butanol, ethyl acetate and hexane were used as dissolved solvent for determination $\gamma$-oryzanol content an increase temperature from 27 to $180{ }^{\circ} \mathrm{c}$. The amount of $\gamma$-oryzanol did not vary significantly with temperature lower than $120{ }^{\circ} \mathrm{c}$. For $\gamma$-oryzanol concentration, a significant change with temperature were higher than $120^{\circ} \mathrm{c}$. However, when hexane was used as dissolved solvent no significant change in the amount of $\gamma$-oryzanol on overall studying temperature.
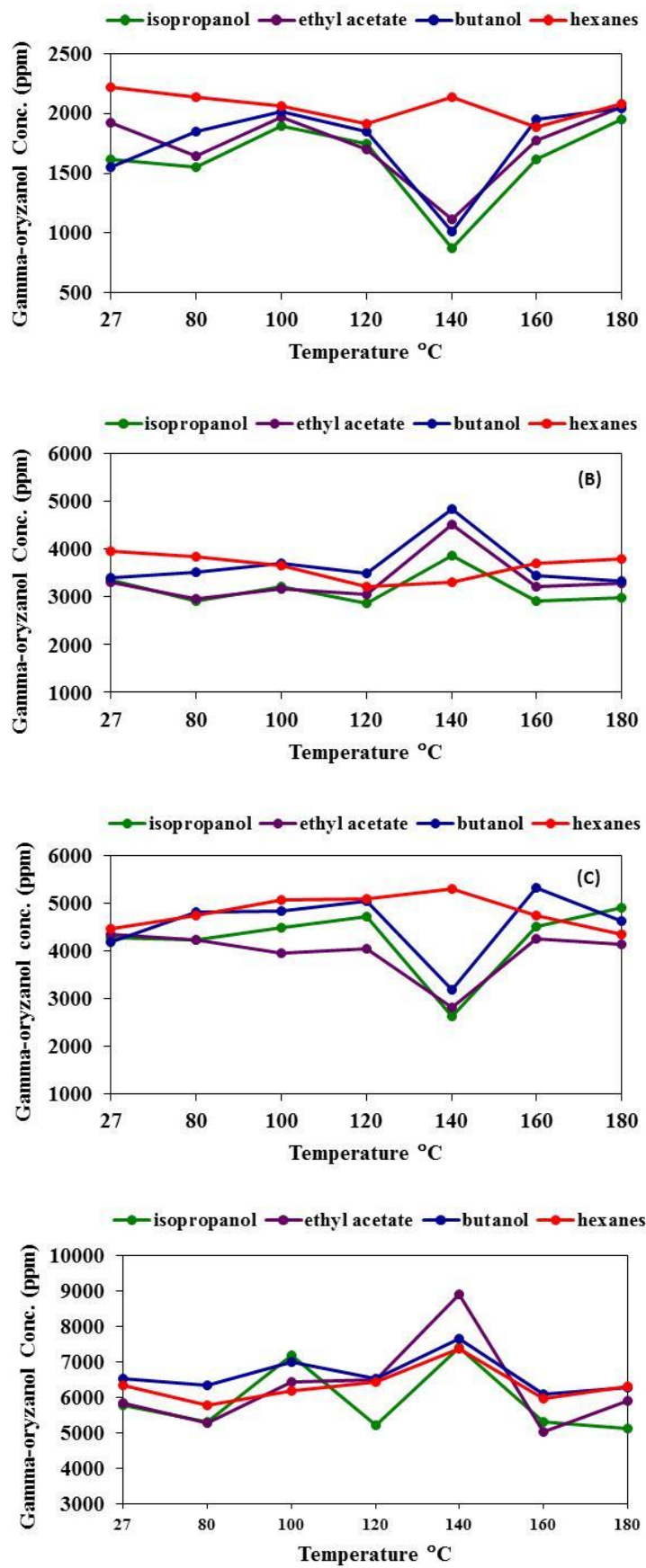

Fig. 2. The relationship between the Gamma-oryzanol concentrations (ppm) with variable temperature of edible commercial $\mathrm{RBO}$ in the different solvent analyzation for the brands King (A) Chim (B) King (high oryzanol) (C) and Alpha 1 (D).

These results can be described by the research of Bucci et al., 2003 [16]. They showed the data for using isopropanol and $n$-heptane to dilute the oil samples in determination of $\gamma$-oryzanol in RBO via spectrophotometric. Their results indicated that the total concentration of $\gamma$-oryzanol is determined measuring the absorbance of an oil solution in $n$-heptane $\left(\lambda_{\max }=314 \mathrm{~nm}\right)$ be not negligible and the results inaccurate because of the oil "matrix" is absorbed this wavelength. They suggested the using of isopropanol $\left(\lambda_{\max }=\right.$ $327 \mathrm{~nm}$ ) as solvent to improve the accuracy of the technique. Due to the fact that it is possible to get rid of the interference of the oil matrix. Kkuwijitjaru et al., 2004 [25] reported that the degradation of $\gamma$-oryzanol in RBO could be found at the temperature higher than $120{ }^{\circ} \mathrm{C}$ described by a pseudo-first order kinetics model. Nystrom et al., 2007 [26] found that the good heat stability of RBO has been attributed to its high content of steryl ferulates and tocopherols presents in the oil.

\section{Determination of Acid Value}

Acid value (AV) is considered to be an indicator of oil quality. AV was found to increase with increasing temperature of the oil and depended on $\gamma$-oryzanol content in the oil (see Table II) because of ferulic acid in $\gamma$-oryzanol structure can react with $\mathrm{KOH}$. It was due to the cleavage and oxidation of double bonds to form carbonyl compounds which oxidized to low molecular weight fatty acid during heating [27], [28]. Moreover, low molecular weight fatty acid in the oil may be lost through volatilization during heating [29].

TABLE II: ACID VALUE OF COMMERCIAL RICE BRAN OIL

\begin{tabular}{lccc}
\hline \multirow{2}{*}{ RBO brands } & \multirow{2}{*}{$\begin{array}{c}\text {-oryzanol } \\
\text { label }(\mathrm{ppm})\end{array}$} & \multicolumn{2}{c}{ Acid value $(\mathrm{mg} \mathrm{KOH} / \mathrm{g}$ of sample $)$} \\
\cline { 3 - 4 } & $>2,000$ & 0.22 & RT. $\left(27^{\circ} \mathrm{c}\right)$ \\
\hline King & No label & 0.31 & 0.29 \\
Chim & $>4,000$ & 0.53 & 0.45 \\
King(high & $>5,000$ & 0.60 & 0.58 \\
oryzanol $)$ & & & 1.03 \\
Alpha1 & & & \\
\hline \hline
\end{tabular}

\section{CONCLUSION}

Gamma-oryzanol concentration in edible commercial RBO was changed at high temperature than $120{ }^{\circ} \mathrm{c}$ due to the degradation of $\gamma$-oryzanol in the oil. It is presumed to a case of acid value increasing at high temperature from reacting of the ferulic acid in $\gamma$-oryzanol with $\mathrm{KOH}$. For the determination of $\gamma$-oryzanol in RBO, isopropanol was recommended for accuracy solvent in determination $\gamma$-oryzanol.

\section{ACKNOWLEDGMENT}

The authors wish to thank Department of Chemistry, Faculty of Science Maejo University, Chiang Mai, Thailand for financial support.

\section{REFERENCES}

[1] M. A. da Siva, C. Sanches, and E. R. Amante, "Prevention of hydrolytic rancidity in rice bran," Journal of Food Engineering, vol. 75, pp. 487-491, 2006

[2] V. R. Pestana, R. C. Zambiazi, C. R. B. Mendoca, M. H. Bruscatto, M J. Lerma-Garcia, and G. Ramis-Ramos, "Quality changes and tocopherols and $\gamma$-oryzanol concentrations in RBO during the refining process," Journal of the American Oil Chemists' Society, vol. 85, pp. 113-119, 2008.

[3] V. V. Hoed, G. Depaemelaere, J. V. Ayala, P. Santiwattana, R. Verhr, and W. D. Greyt, "Influence of chemical refining on the major and 
minor components of rice bran oil," Journal of the American Oil Chemists' Society, vol. 83, pp. 315-321, 2006.

[4] R. Cheruvanky, "Bioactives in rice bran and rice bran oil," in Phytochemical s as Bioactive Agents, W. R. Bidlack, S. T. Omaye, M. S. Meskin, D. K. W. Tophan, Eds. Florida: CRC Press:, 2000, pp. 213-240.

[5] C. J. Bergman and Z. Xu, "Genotyoe and environment effects on tocopherols, tocotrienola and gamma-oryzanol contents of southern US rice," Cereal Chemistry Journal, vol. 40, pp. 446-449, 2003.

[6] V. Piironen, D. G. Lindsay, T. A. Miettinen, J. Toivo, and A. Lampi, "Review: Plantsterols: Biosynthesis, biological function and their importance to human nutrition," Journal of the Science and Agricultural, vol. 80, pp. 939-966, 2000.

[7] Y. Akiyama, K. Hori, T. Takahashi, and Y. Yoshiki, "Free radical scavenging activities of $\gamma$-oryzanol constituents," Food Science and Technology Research, vol. 11, pp. 296-297, 2005.

[8] A. Berger, D. Rein, A. Schafer, I. Monnard, G. Gremaud, P. Lambelet, and C. Bertoli, "Similar cholesterol-lowering properties of ricebran oil, with carried $\gamma$-oryzanol in mildly hypercholesterolemic men," European Journal of Nutrition, vol. 44, pp. 163-173, 2005.

[9] T. A. Wilson, R. J. Nicolosi, and D. Woolfrey, "Rice bran oil and oryzanol reduce plasma lipid and lipoprotein cholesterol concentrations and aortic cholesterol ester accumulation to a greater exten than ferulic acid in hypercholesterolemic hamsters," The Journal of Nutrition Biochemistry, vol. 18, pp. 105-112, 2007.

[10] T. Akihisa, K. Yasukawa, T. Tamaura, M. Ukiya, Y. Kimura, N. Shimizu, and M. Takido, "Triterene alcohol and sterol ferulates from rice bran and their anti-inflammatory effects," Journal of Agricultural and Food Chemistry, vol. 48, pp. 2313-2319, 2000.

[11] K. Yasukawa, T. Akihisa, Y. Kimura, T. Tamaura, and M. Takido, "Inhibitory effect of cycloartenol ferulate, a component of rice bran, on tumor promotion in two stage carcinogenesis in mouse skin," Biological \& Pharmaceutical Bulletin, vol. 21, pp. 1072-1076, 1998.

[12] M. Ismali, G. AL-Naqeeb, W. A. A. Mamat, and Z. Ahmad, "Gamma-oryzanol rich fraction regulates the expression of antioxidant and oxidative stress related genes in stressed rat's liver," Nutrition \& Metabolism, vol. 7, pp. 7-13, 2010.

[13] C. Juliano, M. Cossu, M. C. Alamanni, and L. Piu, "Antioxidant activity of gamma-oryzanol: Mechanism of action and its effect on oxidative stability of pharmaceutical oils," International Journal of Pharmaceutics, vol. 299, pp. 146-154, 2005.

[14] J.-S. Kim and J. S. Godber, "Oxidative stability and vitamin E levels increased in restructured beef roast with added rice bran oil," Journal of Food Quality, vol.24, pp. 17-26, 2001.

[15] J. N. Nanua, J. U. McGregor, and J. S. Godber, "Influence of high-oryzanol rice bran oil on the oxidative stability of whole milk power," Journal of Dairy Science, vol. 83 pp. 2426-2431, 2000

[16] R. Bucci, A. D. Magri, A. L. Magri, and F. Marini, "Comparision of three spectrophotometric methods for the determination of $\gamma$-oryzanol in rice bran oil," Analytical and Bioanalytical Chemistry, vol. 375, pp. 1254-1259, 2003.

[17] B. M. W. P. K. Amarasinghe, M. P. M. Kumarasiri, and N. C. Gangodavilage, "Effect of method of stabilization on aqueous extraction of rice bran oil," Food and Bioproducts Processing, vol. 87, pp. 108-114, 2009.

[18] S. Lilitchan, C. Tangprawat, K. Ayusuk, S. Krisnakgkura, S. Chokmoh, and K. Krisnangkura, "Partial extraction method for the rapid analysis of total lipids and $\gamma$-oryzanol contents in rice bran," Food Chemistry, vol. 106, pp. 752-759, 2008.
[19] A. Proctor and D. J. Bowen," Ambient-temperature extraction of rice bran oil with hexane and isopropanol," Journal of the American Oil Chemists' Society, vol. 73, pp. 811-813, 1996.

[20] A. Proctor, V. M. Jackson, M. Scott, and P. K. Clark, "Rapid equilibrium extraction of rice bran oil at ambient temperature," Journal of the American Oil Chemists 'Society, vol. 71, pp. 1295-1296, 1994.

[21] W. Hu, J. H. Well, T.-S. Shin, and J. S. Godber, "Conparision of isopropanol and hexane for extraction of vitamin $\mathrm{E}$ and oryzanols from stabilized ricr bran," Journal of the American Oil Chemists' Society, vol. 73, pp. 1653-1656, 1996.

[22] M.-H. Chen and C. J. Bergman, "A rapid procedure for analyzing rice bran tocopherol, tocotrienol and $\gamma$-oryzanol contents," Journal of Food Composition and Analysis, vol. 18, pp. 139-152, 2005.

[23] I. G. Zingoneanu, L. Williams, Z. Xu, and C. M. Sabliov, "Determination of antioxidant components in rice bran oil extracted by microwave-assisted method," Bioresource Technology, vol. 99, pp. 4910-4918, 2008.

[24] X. Qiao, T.-S. Chung, W. F. Guo, T. Matsuura, and M. M. Teoh, "Dehydration of isopropanol and its comparison with dehydration of butanol isomers from thermodynamic and molecular aspects," Journal of Membrane Science, vol. 252, pp. 37-49, 2005.

[25] P. Khuwijitjaru, N. Taengtieng, and S. Changprasit, "Degradation of famma-oryzanol in rice bran oil during heating: An analysis using derivative UV-spectrophotometry," Silpakorn University International Journal, vol. 4, pp. 154-165, 2004.

[26] L. Nystrom, T. Achrenius, A. Lampi, R. A. Moreau, and V. Piironen, "A comparison of the antioxidant properties of steryl ferulates with tocopherol at high temperatures," Food Chemistry, vol. 101, pp. 947-954, 2007.

[27] J. Irwandi and Y. B. Cheman, "Effect of rosemary and sage extract on frying performance of RBD palm olein during deep fat frying," Food Chemistry, vol. 69, pp. 301-308, 2000

[28] M. F. Ramadan, M. M. Afify Amer, and M. S. Abd El-Rahman, "Correlation between physicochemical analysis and radical scavenging activity of vegetable oil blends as affected by frying of French fries," European Journal of Lipid Science and Technology, vol. 108, pp. 670-678, 2006.

[29] M. S. Abd El-Rahman, E. A. Makhzangy, and M. F. Ramadan, "Antiradical performance \& physicochemical characteristic of vegetable oils upon frying of French fries: a preliminary comparative study," Journal of Food Lipids, vol. 13, pp. 259-276, 2006.

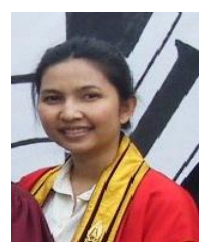

Anakhaorn Srisaipet was born in Thailand on 12 June 1977 and received her degree of doctoral of phillsophy (biochemical technology), at the School of Bioresources and Technology, King Mongkut's University of Technology Thonburi, Bangkok, Thailand in 2007.

Nowadays, she is a lecturer at the Department of chemistry Faculty of Science Maejo University, Chiang Mai, Thailand. The fields of her interested research are lipid technology and enzyme technology.

Mayurachat Nuddagul was born in Thailand and received the bachelor degree of science (chemistry) from the Department of Chemistry, Faculty of Science Maejo University, Chiang Mai, Thailand. 